\title{
EAS hadronic component as registered by a neutron monitor
}

\author{
Yu. V. Balabin ${ }^{1}$, B. B. Gvozdevsky ${ }^{1}$, E. V. Vashenyuk ${ }^{1}$, and D. D. Dzhappuev ${ }^{2}$ \\ ${ }^{1}$ Polar Geophysical Institute (PGI) of RAS, Apatity, Russia \\ ${ }^{2}$ Institute for Nuclear Research of RAS, Baksan Neutrino Observatory, Russia
}

Received: 11 November 2010 - Revised: 10 October 2011 - Accepted: 18 October 2011 - Published: 23 November 2011

\begin{abstract}
For the purposes of a study of hadron component of an extended air shower (EAS), two detectors of cosmic rays (CR) were joined at the Baksan Neutrino Observatory. They are an EAS array detector (the "Carpet") and a neutron monitor (NM). Due to the versatility of a new recording system a master-pulse, which comes from the "Carpet" and marks to occurring of EAS, is joined to NM data. After processing of the huge data set it is found that the time of EAS influence on NM is less than $1 \mathrm{~ms}$. The distribution of time intervals between pulses in the first millisecond after EAS onset is essentially different from the background and shows presence of two processes with characteristic times 45 and $230 \mu \mathrm{s}$.
\end{abstract}

\section{Introduction}

Two cosmic rays detectors, the "Carpet" and the NM have been connected at the Baksan neutrino observatory located in the Northern Caucasus at an altitude of $1700 \mathrm{~m}$ at sea level. Due to the adaptability of a new high-speed data collection system, it has become possible to bind the master-pulse from the "Carpet", indicating the arrival of an EAS, to the NM pulses. A more detailed description of the data collecting system is given in Balabin et al. (2009, 2010). It should be pointed out that the "Carpet" detects the EAS electronphoton component, whereas the NM detects hadrons (neutrons, protons and pions) with energy $>50 \mathrm{MeV}$ (Dorman, 1975). The "Carpet" and the NM, with effective areas of $200 \mathrm{~m}^{2}$ and $2 \times 3 \mathrm{~m}^{2}$, respectively, are placed in the same building, separated by about $15 \mathrm{~m}$. Owing to this combination of instruments with high time precision, we achieve improved accuracy in studying the EAS hadron component.

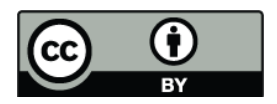

Correspondence to: Yu. V. Balabin (balabin@pgia.ru)

\section{EAS events detection in NM}

The neutron monitor (NM) operated at the Baksan station consists of 6 tubes (channels). In the recording system there is an additional channel (the 7th), which receives the masterpulses from the "Carpet". These pulses are generated at the moment an EAS, produced by the primary particles whose threshold energy is over $6 \cdot 10^{12} \mathrm{eV}\left(N_{e}=10^{5}-10^{7}\right.$ particles), falls down on the "Carpet". Thus, there is an opportunity to study a hadron flux produced by an EAS, which is detected in the NM. The average count rate in Channel 7 (EAS occurrence) is $\sim 80 \mathrm{~min}^{-1}$. In the course of the subsequent processing, it is possible not only to distinguish the pulses from an EAS, but also to study the sequence of the NM-detected pulses following either immediately after the EAS, or some time later.

The algorithm of data processing is as follows. As soon as an EAS pulse is detected, an operating time window $T_{w}$ is opened. The NM pulses are counted and the pulse-to-pulse intervals occurring within this time window are recorded. Then, after a period of time $T_{p}$, the time window of the same duration $T_{w}$, is opened again for reference (background) counting. The preliminary data processing ( $\sim 30-40$ days $)$ found that the probable influence of an EAS on the NM lasts about $1 \mathrm{~ms}$. Based on these, we use $T_{w}=1 \mathrm{~ms}$, and $T_{p}=25 \mathrm{~ms}$.

The first and the most important stage in the data processing is the determination of a distribution of time intervals (DOTI). To determine DOTI we should count the number of cases when the pulse-to-pulse interval (the intervals between successive pulses within the NM) was equal to $t \mu \mathrm{s}$. The type of DOTI indicates the information on the nature of the processes occurring in the NM. Figure 1(a) shows the different kinds of DOTIs obtained from the complete 2009-10 data set. The choice of the value of $T_{p}$ was determined by the condition that in the NM there should be no neutrons from the previous EAS. An increase of the time window $T_{w}$ to over

Published by Copernicus Publications on behalf of the Arbeitsgemeinschaft Extraterrestrische Forschung e.V. 

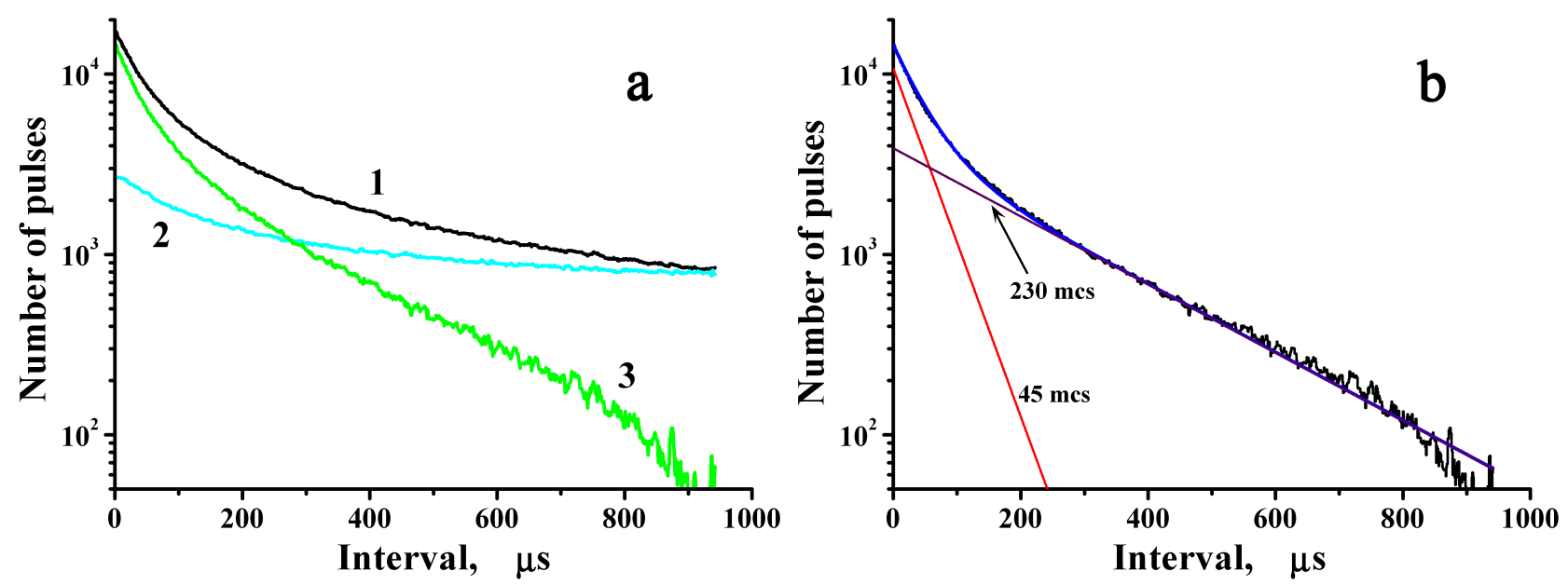

Fig. 1. (a) the distribution of time intervals, which were calculated according to the algorithm. 1) in the operating time window (after EAS), 2) in the reference time window, 3) the difference between 1) and 2); (b) the difference (black line) and a fitting function (blue line). The fitting function consists of two exponentials with characteristic times $\tau_{1}=45$ and $\tau_{2}=230 \mu$ s (red and magenta lines).

$1000 \mu$ s gives no noticeable effect: the DOTI immediately after the EAS arrival (operating DOTI) and the reference DOTI merge. It is clearly seen in Fig. 1(a) where we present DOTI for 100 days. The authenticity of the "Carpet" pulses, came on the recoding system, was also checked. The check was based on the fact that the true pulses from the "Carpet" have a strictly fixed duration of $50 \mu$ s. With the accuracy of recording of $1 \mu \mathrm{s}$, it is easy to determine the pulse duration from the "Carpet".

It is seen from the Fig. 1(a) that the number of short intervals (up to $200-300 \mu$ s) recorded in the operating window is several times as many as the number of short intervals in the reference time window. However, at $t \sim 1000 \mu \mathrm{s}$, their number is becoming equal. Thus, the first result is that the total time of an EAS influence on the NM does not exceed $1000 \mu$ s. This value disagrees with that obtained, for instance, in Stenkin (2008). These studies, however, differ in the observed energy ranges. The NM detects hadrons of at least $50 \mathrm{MeV}$ in energy whereas Stenkin (2008) studied EAS thermal neutrons.

Both the background cosmic rays $(\mathrm{CR})$ and the EAS events obey Poisson law:

$P_{k}(\Delta t)=\frac{\left(N_{0} \cdot \Delta t\right)^{k}}{k !} \exp \left(-N_{0} \cdot \Delta t\right)$

where $\Delta t$ is the time interval, $N_{0}$ is an average number of pulses per time unit, $k$ is a number of detected pulses, $P_{k}(\Delta t)$ is the probability of getting exactly $k$ pulses during the time interval $\Delta t$. An important feature of the Poisson distribution is that if the probability of the pulse number is described by Eq. (1), the probability of the interval value between pulses $\Delta t$ is given by

$w(\Delta t)=N_{0} \exp \left(-\frac{\Delta t}{\tau_{0}}\right)$ where $w(\Delta t)$ is the probability to get an interval $\Delta t$ between pulses, $\tau_{0}$ is a mean interval between pulses (the characteristic time $\left.\tau_{0}=1 / N_{0}\right)$ (Goldansky et al., 1959).

The Poisson distribution also has another feature (Goldansky et al., 1959): the sum of two Poisson processes with the average intensity $N_{1}$ and $N_{2}$ respectively, will be the Poisson distribution with intensity $N_{\text {tot }}=N_{1}+N_{2}$. Hence, the DOTI function $w_{\text {tot }}(\Delta t)$ for the sum of two Poisson processes will be described as Eq. (2) where $\tau_{\text {tot }}=1 / N_{\text {tot }}$, rather than the sum of two exponentials with characteristic times $\tau_{1}=1 / N_{1}$ and $\tau_{2}=1 / N_{2}$. However, while the probability of two Poisson processes' contributions to the total flux is different in the DOTI, the true exponential dependences $w_{1}(t)$ and $w_{2}(t)$ will be observed separately, which correspond to the initial processes $N_{1}$ and $N_{2}$ and characteristic times $\tau_{1}=1 / N_{1}$ and $\tau_{2}=1 / N_{2}$. As Fig. 1 shows the DOTIs in a semilog scale, the exponential curve is shown as a straight line. The DOTI nonlinearity in the chosen scale indicates that process under study is a complicated sum of Poisson processes (Goldansky et al., 1959).

The primary CR energies to which the "Carpet" and the NM respond are different, and in this sense the intensities recorded by these instruments are independent. Thus, in the count rate of an NM we have a complicated sum of Poisson processes. The reference DOTI is calculated only on the background CR fluxes, whereas the operating DOTI are the sum of the CR flux and the EAS. Hence, it is possible to write:

$w(\Delta t)=w_{\mathrm{EAS}}(\Delta t)+W_{C R}(\Delta t)$

where $w(t)$ is the function presenting the operating DOTI, $w_{\text {EAS }}(t)$ is the function of the DOTI from EAS and $w_{C R}(t)$ is the reference DOTI. We see that the operating DOTI is made of the sum of two functions presenting the EAS proper 
and the reference one. One can give the $w_{\mathrm{EAS}}(t)$ as the difference between $w(t)$ and $w_{C R}(t)$. In Fig. 1(a), the difference is shown as a green line and a deviation of the DOTI from the linearity is clearly seen. In Fig. 1(b), the approximation of the difference is shown as the sum of two exponentials. The values of the characteristic times are $\tau_{1}=45 \mu \mathrm{s}$ and $\tau_{2}=230 \mu \mathrm{s}$ and the approximating function is

$w_{\mathrm{EAS}}(\Delta t)=A \exp \left(-\frac{\Delta t}{\tau_{1}}\right)+B \exp \left(-\frac{\Delta t}{\tau_{2}}\right)$

These two exponentials are evidence of the complicated sum of the Poisson processes taking part in the process caused by EAS. The complicated sum points on the two independent processes, which have effects on NM during first $1000 \mu \mathrm{s}$ after an EAS.

\section{EAS-induced multiplicity events in NM}

The papers Balabin et al. $(2010,2011)$ gave a detailed survey of all the multiplicity events detected in a NM in general. In this work we study the NM multiplicities related to EAS only. It is well known that the multiplicity event (ME) is the number of neutrons $M$ detected in the NM over a short period of time. We give below the conditions for search and selection of multiplicity events.

1) before a ME there should be a time interval of at least $T_{\mathrm{pau}}$, during which there are no pulses;

2) the intervals between pulses following each other (after $\left.T_{\text {pau }}\right)$ should not exceed the value $T_{0}$. Total number of pulses in such cluster is ME of $M$. The total duration of the clusters depends on the number $M$. The first interval of more than $T_{0}$ duration finalizes an event.

3) it is necessary that the multiplicity event is associated with the pulse from the "Carpet".

The papers Balabin et al. (2010, 2011) use Criteria 1) and 2). Criterion 3) is introduced in the present work. In Balabin et al. (2011) optimal values to be used in search of MEs have been found: $T_{0}=500 \mu$ s and $T_{\text {pau }}=5000 \mu$ s. The same values are used in the present study. In data processing, both the search of all MEs (Criteria 1) and 2) only) and the MEs accompanied by EAS were used. The ratio between the MEs (see Fig. 2) occurring immediately after the EAS arrival, and the total number of MEs of each number is calculated. We see that MEs with $M>20$ mainly occur immediately after the EAS arrival, and with $M>25$ this ratio is close to 1 , i.e. practically all the events with $M>25$ are induced by an EAS. It is probable that all the MEs with $M<25$ originate from EAS too, but due to spacing between the "Carpet" and the NM small EAS can be omitted by the "Carpet". Because of the small dimensions of the small EASes, they cannot overlap simultaneously both the NM and the "Carpet".

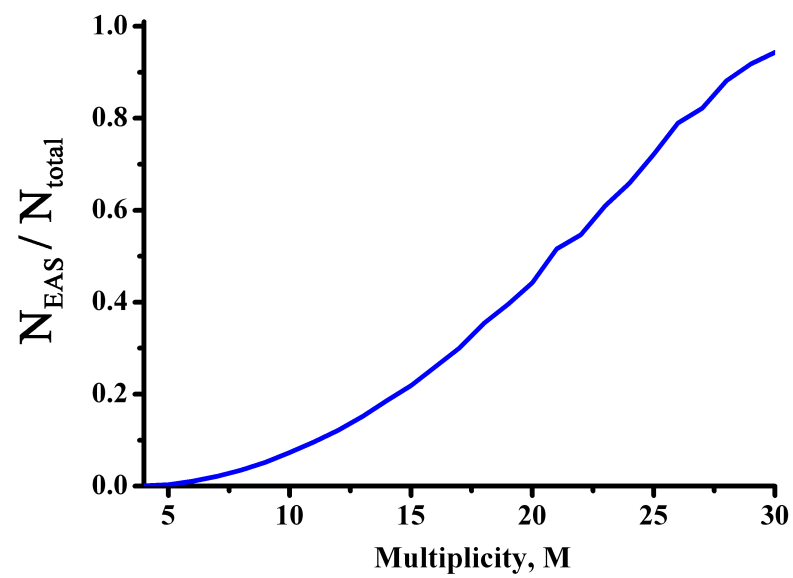

Fig. 2. Ratio $N_{\text {EAS }} / N_{\text {total }}$ on the NM. It is easy to see that large $M$ events are usually accompanied by EAS.

The amount of MEs up to $M=30$ is enough for the DOTI to be calculated with a small statistical error for any given $M$. The procedure is simple. In all the events of the given $M$, the number of intervals of duration $t \mu$ s is counted. Figure 3(c) shows that the DOTI is quite different from the exponential which was calculated for the Poisson process (2). All the DOTI can be fitted by the sum of two exponentials, like in Eq. (4), with characteristic times $\tau_{3}$ and $\tau_{4}$. A study has been carried out to determine in what particular part of ME the processes $N_{3}$ and $N_{4}$ occur. It has been found in Balabin et al. $(2010,2011)$ that the average time intervals in ME don't significantly vary at the initial phase of events. But closer to the end (the final phase) of the events the time intervals increase steadily. The boundary between the initial and final phases is on around 7-10 pulses before end. Based on these results, we have calculated DOTI for the first 10 and for the last 7 intervals separately. All three DOTI for $M=25$ are shown in Fig. 3. The DOTI based on the entire set of events is a sum of exponentials with $\tau_{3}=35 \mu \mathrm{s}$ and with $\tau_{4}=165 \mu \mathrm{s}$ (Fig. 3(c)), whereas the DOTI based on the first 10 and the last 7 intervals consist of practically one exponential with each (Fig. 3(a),(b)), having characteristic times $\tau_{3}=35 \mu \mathrm{s}$ and $\tau_{4}=165 \mu$ s respectively. This is direct proof of the presence of two different processes in one ME, with one of these forming the initial phase, and the other the final or relaxation phase. Similar result is also obtained with $M<20$.

However, while the DOTI for the relaxation part preserves its linearity, the DOTI for the initial part, as $M$ decreases, increasingly gets a shape like in Fig. 3(c), i.e. loses its specificity. What's more, it makes no difference whether the MEsbased DOTI have been found immediately after the EAS arrival or whether it is based on the whole set of MEs. The detected property is specific for all MEs. The obtained result confirms the assumption stated in Balabin et al. $(2010,2011)$ that large MEs consist of two phases - the initial and relaxation phase, which differ in their characteristic time. The re- 


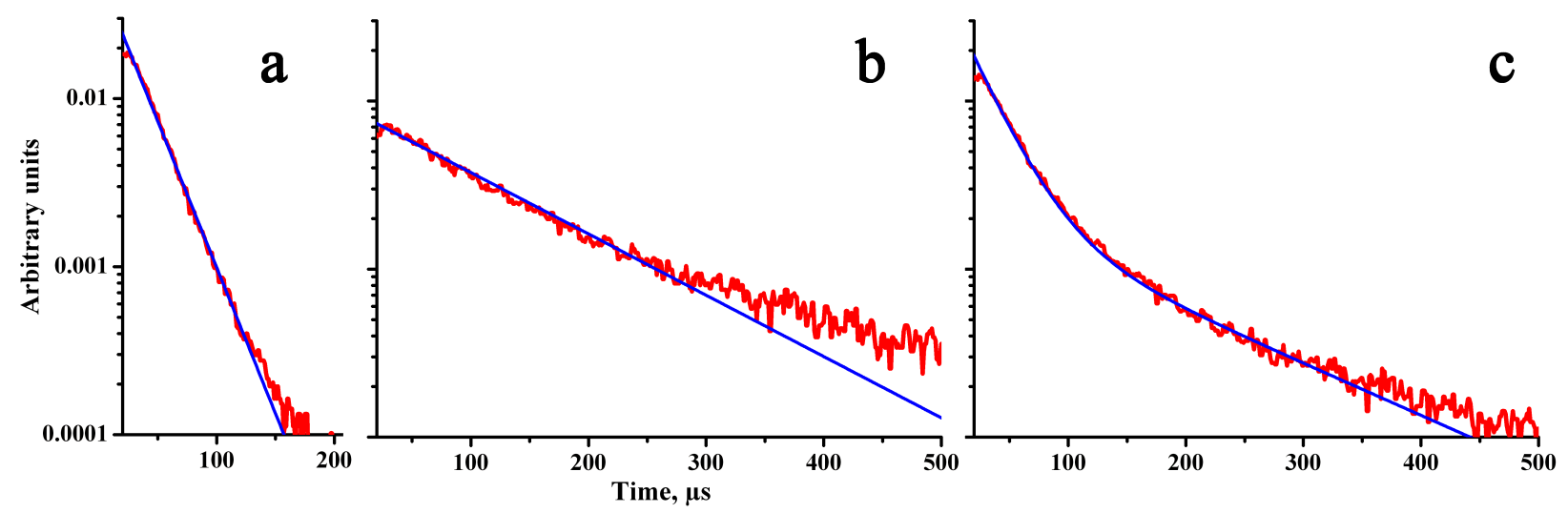

Fig. 3. Distribution of time intervals between pulses (DOTI) is made for $M=25$. (a) the DOTI for the 10 initial intervals of MEs only; (b) the DOTI for the 7 last intervals of MEs only; (c) the total DOTI (all intervals of MEs are used). Fitting functions are shown as a blue line: they are simple exponential functions for (a) and (b), there is a sum of them for (c). Arbitrary units are normalized for a total number of pulse.

laxation phase is composed of the last $7-8$ pulses in a $\mathrm{ME}$, and the initial phase is composed of the first $M-10$ pulses. For the events with $M<10$, separation ME into phases is difficult. Besides, the calculated values of times $\tau_{3}$ and $\tau_{4}$ are close to the characteristic times $\tau_{1}$ and $\tau_{2}$, respectively, which describe the DOTI immediately after the EAS arrival (Fig. 1(b)). The differences between the time intervals are explained by the fact that $\tau_{3}$ and $\tau_{4}$ are calculated for a particular value of $M$ whereas the DOTI immediately after the EAS arrival is based on all possible values of $M$.

\section{Conclusions}

We have studied the distribution of NM pulses calculated immediately after the EAS master-pulse arrival. The similar distribution of the reference time intervals outside EAS influence zone has been compared. We have found that the hadron component of the EAS influences a conventional NM for not over $1 \mathrm{~ms}$ after the EAS arrival. The calculation of the DOTI between the pulses (within $1 \mathrm{~ms}$ after the EAS arrival) has shown the presence of a sequence of the NM pulses produced by high-energy hadrons detected in the NM during the EAS influence. The average lifetime of the particle population inside the NM is $45 \mu \mathrm{s}$.

We found that the ratio between the multiplicity events accompanied by EAS and their total number increases as $M$ increases, achieving 1 for large multiplicities $(M>25)$. The calculation of the DOTI separately for the initial and final parts of the MEs shows that the MEs for $M>10$ consist of two different processes having their own characteristic times, with there being no difference in whether the ME is accompanied by an EAS or not.
Acknowledgements. This work was supported by the Russian Foundation for Basic Researches Grant No 09-02-00076-a and the Program of the Presidium of the Russian Academy of Sciences No 8 "Physics of Neutrino and Neutrino Astrophysics".

Edited by: T. Laitinen

Reviewed by: J. Hörandel and two other anonymous referees

\section{References}

Balabin, Yu. V., Dzhappuev, D. D., Gvozdevsky, B. B., Maurchev, E. A., Kudzhaev, A. U., and Mikhailova, O. I.: Neutron Multiplicity: Local Hadronic Showers and Extensive Air Showers, Bull. Russ. Acad. Sci. Phys., 75, 364-366, 2011.

Balabin, Yu. V., Gvozdevsky, B. B., Vashenyuk. E. V., and Schur, L. I.: Dynamics of relativistic SCRs and registration of multiple neutrons during the event of December 13, 2006, Bull. Russ. Acad. Sci.: Physics series, 73, 304, 2009.

Balabin, Yu. V., Vashenyuk, E. V., Dzhappuev, D. D., Gvozdevsky, B. B., Maurchev, E. A., Kudzhaev, A. U., and Mikhailova, O. I.: Searching of local atmospheric shower on neutron monitors, in: Proc. 31st Russian CR Conf., 5-9 July 2010, Moscow, 2010.

Dorman, L. I.: Experimental and theoretical fundamental astrophysics of cosmic rays, Moscow, NAUKA, 464, 1975 (in Russian).

Goldansky, V. I., Kutsenko, A. V., and Podgoretsky, M. I.: Counter statistics at nuclear particle registration, Fizmatgiz, Moscow, 340, 1959 (in Russian).

Stenkin, Yu. V.: Thermal neutrons in EAS: a new dimension in EAS study, Nucl. Phys. B, 175-176, 326, 2008. 